\title{
Grand challenges in high energy and astroparticle physics
}

\author{
J. W. F. Valle ${ }^{1 *}$ and Jan de Boer ${ }^{2}$ \\ ${ }^{1}$ Astroparticle and High Energy Physics Group, Consejo Superior de Investigaciones Cientificas, Paterna, Valencia, Spain \\ ${ }^{2}$ Faculty of Science, Institute for Theoretical Physics, University of Amsterdam, Amsterdam, Netherlands \\ *Correspondence: valle@ific.uv.es \\ Edited by: \\ Emilio Elizalde, Consejo Superior de Investigaciones Cientificas, Spain \\ Reviewed by: \\ Emilio Elizalde, Consejo Superior de Investigaciones Científicas, Spain
}

\section{OVERVIEW}

The field of High Energy and Astroparticle Physics aims at unraveling the laws of physics at the shortest distance scales and how these give rise to the universe we live in. Up to the highest energies that can presently be created by man-made accelerators, nature is remarkably well described by a single theory, the standard model of particle physics. The electromagnetic, weak and strong interactions among the elementary fermions are well described by a quantum field theory merging quantum mechanics and special relativity, and incorporating the principle of gauge symmetry. In the Standard Model of particle physics the basic forces other than gravity are mediated by the exchange of intermediate vector bosons associated to the standard model gauge symmetry: the photon, the gluons and the electroweak bosons. The theoretical formulation of the Standard Model was developed from the mid to late 20th century, and its current form has gained general acceptance after the experimental confirmation of the existence of quarks in the mid 1970s. The gauge bosons associated to the electroweak gauge symmetry, the photon and $\mathrm{W}$ and $\mathrm{Z}$ gauge bosons, were directly produced for the first time at CERN in 1983, while the gluons are associated to the $\mathrm{SU}(3)$ color symmetry and were already discovered in the late 1970s. Today we know for certain that there are at least three types or "generations" of elementary constituents of matter. The simplest way to provide masses for the $\mathrm{W}$ and $\mathrm{Z}$ bosons as well as for the charged fermions is to spontaneously break the gauge symmetry down to the color and electromagnetic subgroup. This suggests the existence of a physical elementary scalar particle, the socalled Higgs boson, which is indeed part of the Standard Model, and whose recent discovery at the Large Hadron Collider (LHC) experiment at CERN by the ATLAS (see Figure 1) and CMS Collaborations constitutes an outstanding achievement in particle physics, and a triumph for theory.

The recent experimental confirmation of the existence of the Higgs boson has put the Standard Model on a very firm footing. However, there are several reasons why the Standard Model is believed to be incomplete and even within the Standard Model many puzzles remain. One of these puzzles, for example, involves the neutrino sector. Among the elementary constituents of matter neutrinos are unique in that they do not carry electric charge and as a result they undergo only weak interactions, hence their experimental elusiveness: neutrinos may pass through ordinary matter almost unaffected. There is one neutrino "flavor" associated to each generation of elementary constituents. Understanding flavor from first principles remains a mystery, and it is a great challenge to find a natural explanation for both the origin as well as the values of the many free parameters that characterize the flavor sector. As unique and fundamental building blocks of the Standard Model neutrinos may hold important clues for what lies ahead.

Given the success of the Standard Model and the gauge principle on which it is based, it is now widely believed that any extension of the Standard Model, even ones that incorporate gravity, should be based on a gauge principle of some sort. Unfortunately, it is hard to directly probe the beyond the Standard Model regime. The universe, on the other hand, is capable of accelerating particles to much higher energies than humans can, and are a great potential source of information. But the precise mechanism by which nature manages to create very highly energetic cosmic rays remains poorly understood.

On the other side of the extreme, there is yet another theory which is remarkably successful, the standard model of cosmology. It describes several aspects of cosmology very well and recent results from the Planck satellite (see Figure 1) have yet again confirmed its predictions. It raises however at least as many questions as it answers. It assumes the existence of dark matter and dark energy, neither of which are part of the standard model. It assumes a period of inflation in the early universe which is also very difficult to reconcile with the standard model. And it assumes that the universe started out in a singular configuration, the famous big bang.

A proper description of the big bang requires a theory which reconciles general relativity with quantum mechanics. String theory is a theory which does precisely that, and has provided a plethora of theoretical ideas over the past decades, but which remains extremely difficult to verify experimentally.

Progress in all these fields will require great effort both on the theoretical as well as the experimental side. Precise astrophysical measurements, combined with earth based experimental data, must be developed side by side with theoretical ideas to further improve the understanding of the world we live in.

Let us first mention some of the challenges facing particle physics today:

- Can nature be described by a unified theory where all forces have a common origin? The electromagnetic, weak and strong forces appear to unify somewhat below the Planck scale within unified extensions of the Standard Model. In addition one expects that also gravityunifies at an even higher energy 


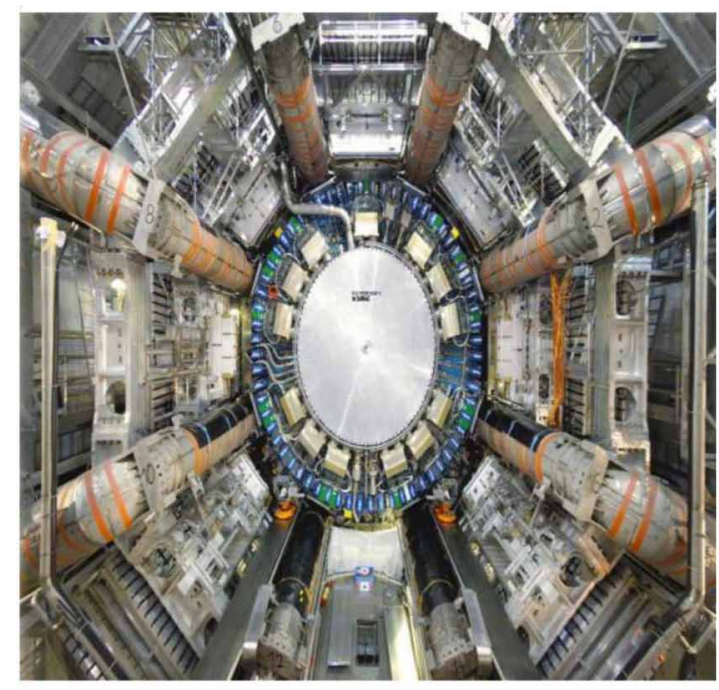

The ATLAS detector at CERN

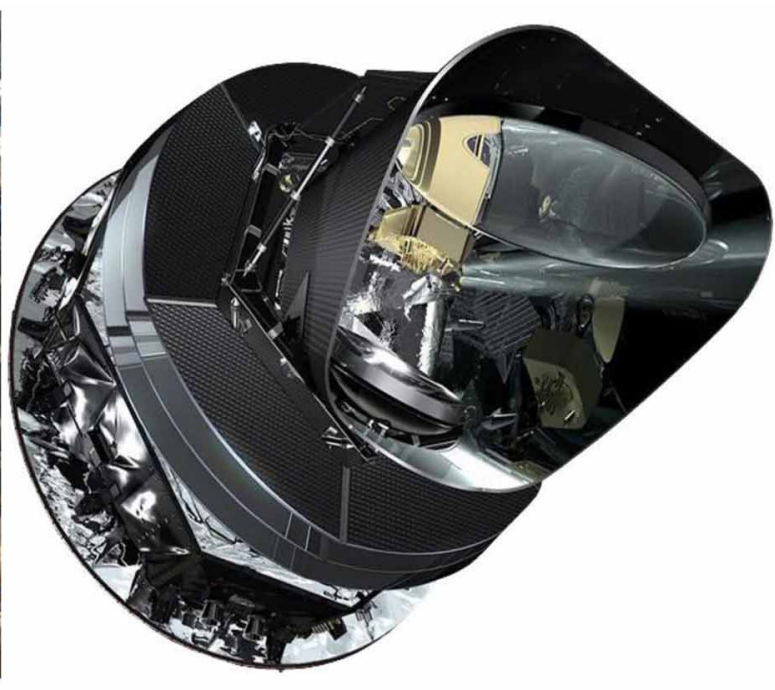

The Planck satellite.

FIGURE 1 | Illustrative views of the ATLAS detector and Planck satellite instrument.

scale with the other forces. Unification would provide an elegant way to explain the fundamental interactions in nature.

- The mass scale of the fundamental particles is determined by the scale at which one of the symmetries of fundamental interactions, the electroweak symmetry, is broken. This happens spontaneously due to the Higgs field in the vacuum. This picture, incorporated in the Standard Model, has recently been strengthened by the discovery of the Higgs boson at the LHC. However it raises a consistency puzzle associated with the small value of the Higgs field in the vacuum when compared with the fundamental Planck scale that characterizes gravity. One approach to the problem of explaining this scale difference involves a new symmetry of nature-supersymmetry-which has so far not been vindicated by experiment.

- Why do we see three families of particles with so different masses? The masses of fundamental particles are well measured by experiments. For the case of the charged leptons, we have three fundamental particles: electrons, muons and taus. They have the same quantum numbers (properties) but their masses are quite different. Similarly the large top quark mass seems to suggest that it plays a special role.
Below some grand challenges will be elaborated upon, but it is impossible to do full justice to this field in this brief overview. An overview that covers many aspects in much more detail can for example be found at the website of the particle data group, http://pdg.lbl.gov/2012/reviews/contents_ sports.html

\section{ASTROPARTICLE PHYSICS: THE STANDARD MODEL AND BEYOND}

There are many unsolved challenges regarding the underlying physics of quarks and leptons, the basic building blocks of matter. Among the elementary constituents of matter neutrinos play a special role as cosmic probes. They come from natural sources such as geological neutrinos from natural radioactivity and solar neutrinos from nuclear fusion inside the Sun. For example, each second, about 65 billion solar neutrinos pass through every square centimeter on the part of the Earth that faces the Sun. Since neutrinos are hardly absorbed by the mass of the Earth, the surface area on the side of the Earth opposite the Sun receives about the same number of neutrinos as the side facing the Sun. There are also atmospheric neutrinos from cosmic ray interactions in the Earth's atmosphere, neutrinos from supernova explosions, etc.
Their energies cover an impressive range all the way from that of cosmological neutrinos primordially produced in the Big Bang, up to that of high energy sources such as active galactic nuclei. The birth of extra solar system neutrino astronomy in the eighties with the detection of neutrinos from SN1987a in the Large Magellanic Cloud has paved the way to a new generation of decisive neutrino oscillation experiments in the nineties which, altogether, brought on a firmer observational basis the long-standing problem of solar neutrinos. The ultimate elucidation of the solar neutrino puzzle had to wait for the confirmation of the oscillation hypothesis by the nuclear reactor experiment KamLAND. This experiment measured not only the flux of antineutrinos from distant nuclear reactors in Japan, but also confirmed the spectrum distortion characteristic of the large mixing angle oscillations. This was important to exclude alternative non-standard solutions, expected in many theoretical models which ascribe the smallness of neutrino mass to the heaviness of some hypothetical particle messengers such as right-handed neutrinos in seesaw-type schemes.

The uniqueness of neutrinos as a fundamental building block of the Standard Model of elementary particle interactions 
suggests that they may hold the clue for what lies ahead our current horizon. Just to mention some of the open issues:

- The origin of neutrino mass: Why are neutrinos so much lighter than the other elementary fermions? Does this hint toward the existence of some sort of unification of the gauge interactions? At what scale does the physics responsible for neutrino masses lie? Does it relate to unification? Could neutrino masses be probed at accelerators like the LHC? Is neutrino mass generation a manifestation of supersymmetry through $\mathrm{R}$ parity violation?

- The nature of neutrinos: Are neutrinos their own anti-particles? Is lepton number violated in nature? The observation of neutrinoless double beta decay would settle this question and great experimental efforts are indeed currently ongoing.

- The pattern of neutrino mixing: Why are lepton mixing angles so different from quark mixing angles? Is there a unified theory of flavor? Is there an underlying flavor symmetry?

- Probing non-standard neutrino interactions: are there lepton flavor violation effects beyond oscillations? How do they relate to those in the charged lepton sector, such as muon-to-electron conversion in nuclei? How do such flavor and/or CP violation processes relate to the neutrino masses and mixing angles? This complementary information will be vital to help sort out the flavor puzzle and the origin of neutrino mass.

\section{COSMOLOGY AND PARTICLE PHYSICS}

Recent progress in probing the anisotropies of the cosmic microwave background (CMB) by the WMAP and Planck space missions provides valuable information on the early Universe, such as its age and other data relevant to understand the mechanism of inflation and the formation of its earliest structures. These experiments provide information about the absolute scale of neutrino mass as well as the effective number of relativistic species. We know that over $80 \%$ of the matter in the universe is not made of atoms but of a yet unknown substance that cannot be directly seen and is hence called dark matter. This follows from astronomical and cosmological observations that reveal the gravitational effect of dark matter on galaxies and other astronomical objects. Since dark matter is expected to be everywhere in the Milky Way even a weak interaction with atoms would lead to an observable signal. Pair annihilation of dark matter particles in the center of the Milky Way would produce cosmic rays that are observable by satellites. Underground experiments could also produce rare interactions of dark matter with atomic nuclei. Moreover, collisions of protons at high energies available at the LHC could lead to the production of such dark matter particles.

- What is dark matter made of? The key problem is that the Standard Model of particle physics cannot explain the origin of this mysterious component of the universe. Most of the matter in the universe is made of a material beyond the understanding of current particle physics theories.

- What is the mechanism responsible for inflation and the explanation of dark energy? Indeed no convincing interpretations of the particle physics origin of inflation and of the dynamics behind the cosmological constant have so far been presented.

- Why is there more matter than antimatter in the universe? The Standard Model of particle physics treats matter and antimatter in essentially the same way. Yet wherever we look in the universe all we see is matter. Why is the universe made of matter instead of antimatter? When in contact, matter and antimatter immediately annihilate into energy. However we exist because there was initially slightly more matter than antimatter, leaving a sufficient amount of matter after annihilation to form our universe.

Due to their weak interaction neutrinos constitute a valuable probe of the most remote past of our Universe, just after the Big-Bang. Indeed this information may shed light on mysteries such as the origin of matter-the baryon asymmetry of the Universe-as well as the particle physics interpretation of dark matter. Indeed models of neutrino mass have been proposed that lead to specific particle physics candidates for dark matter, such as decaying majorons or gravitinos. These require different detection techniques when compared to the more popular WIMP (Weakly interacting massive particle), an alternative suggested by the simplest supersymmetric models.

\section{QUANTUM GRAVITY AND STRING THEORY}

To guide our search for theoretical explanations of the plethora of phenomena described above, a more top down approach could in principle be very helpful. At extremely high energies, the standard model and its extensions necessarily break down and a complete description of physics requires a quantum theory of gravity. To find such a theory and establish its validity beyond a reasonable doubt has often been referred to as one of the holy grails of theoretical physics. String theory is presently the most promising candidate theory of quantum gravity, though with a direct experimental verification still lacking, one should obviously keep an open mind toward other possibilities. Nevertheless, string theory has provided a rich theoretical framework with lasting value no matter what. One of the most amazing achievements of string theory is the so-called AdS/CFT correspondence, which maps strongly coupled field theories in $\mathrm{d}$ dimensions to weakly coupled gravitational theories in $\mathrm{d}+1$ dimensions and vice versa. This correspondence provides a concrete realization of the principle of holography, which all theories of quantum gravity must obey. This AdS/CFT correspondence provides us with a new theoretical tool to study a variety of problems in physics, but it is not intended to be the starting point of a top-down construction of a realistic model of our universe. The search for the latter is a field which is usually referred to as string phenomenology. Some of the key questions in these areas are

- Is there a better description of string theory or M-theory which is non-perturbative? In quantum gravity, is space-time fundamental or an emergent concept? What is the precise theoretical framework that we should use to describe the Big Bang and singularities inside black holes? 
What are the fundamental degrees of freedom that make up black holes in the first place? Does a better understanding of quantum gravity lead to observable predictions for our universe or shed light on the value of the cosmological constant?

- In the AdS/CFT correspondence, how does approximate locality in a theory of gravity arise from the strongly coupled dual theory? Does this locality break down in ways we have not previously anticipated? What field theory properties explain the universal features of gravity? Which phases of strongly coupled matter do have approximate gravitational descriptions and can one classify those?

- Is it possible to provide exact solutions of some of the quantum field theories that appear in the AdS/CFT correspondence, like $\mathrm{N}=4$ super YangMills theory? Do these exact solutions describe all amplitudes and correlation functions? Do these exact solutions shed light on some of the problems mentioned above, like the emergence of an approximately local dual gravitational description?

- To what extent can one construct realistic models of our own universe within string theory? Does this process provide certain generic and experimentally testable predictions? Do such models generically lead to extra dimensions and hidden sectors? Are universes like our own in any way distinguished or are most universes unlike our own? If so, what are the things that one can meaningfully predict? Will we be able to interact with other possible universes?

\section{ACKNOWLEDGMENTS}

Work supported by MINECO grants FPA2011-22975, MULTIDARK
Consolider CSD2009-00064, by Prometeo/ 2009/091 (Gen. Valenciana), by EU ITN UNILHC PITN-GA-2009-237920. This work is part of the research programme of the Foundation for Fundamental Research on Matter (FOM), which is part of the Netherlands Organisation for Scientific Research (NWO).

Received: 14 June 2013; accepted: 28 June 2013; published online: 23 July 2013

Citation: Valle JWF and de Boer J (2013) Grand challenges in high energy and astroparticle physics. Front. Physics 1:5. doi: 10.3389/fphy.2013.00005

This article was submitted to Frontiers in High-Energy and Astroparticle Physics, a specialty of Frontiers in Physics.

Copyright (c) 2013 Valle and de Boer. This is an open-access article distributed under the terms of the Creative Commons Attribution License, which permits use, distribution and reproduction in other forums, provided the original authors and source are credited and subject to any copyright notices concerning any third-party graphics etc. 